\title{
ESTACIONALIDAD DE NACIMIENTOS, MATRIMONIOS Y DEFUNCIONES EN LA REGIÓN MAGALLÁNICA. COMPARACIÓN CON LA ÉPOCA DE LA COLONIZACIÓN
}

\author{
MIGUEL HERNÁNDEZ*, CLARA GARCÍA-MORO* \& MIREIA ESPARZA*
}

\begin{abstract}
RESUMEN
Se ha estudiado la estacionalidad de los nacimientos, matrimonios y defunciones en la región magallánica en el período 1997-2009. Los nacimientos son más frecuentes entre los meses de abril a octubre, mientras que los matrimonios presentan un máximo en verano y las defunciones en invierno. Los resultados actuales muestran diferencias significativas con los de la época de la colonización que pueden explicarse por los cambios socioeconómicos.
\end{abstract}

PALABRAS CLAVE: estacionalidad, Magallanes, tiempo presente, colonización, cambio socioeconómico.

\section{ABSTRACT}

\section{SEASONALITY OF BIRTHS, MARRIAGES AND DEATHS IN THE MAGELLAN REGION. COMPARISON WITH THE TIME OF SETTLEMENT}

The seasonality of births, marriages and deaths in the Magellan region in the period 1997-2009 has been studied. Births are most common between the months of April to October, while marriages have a peak in summer and deaths are more frequent in winter. These results show significant differences with those obtained for the time of settlement which may be explained by socioeconomic changes.

KEY WORDS: seasonality, Magellan, present time, settlement, socioeconomic change.

\footnotetext{
* Sección de Antropología, Departamento de Biología Animal, Facultad de Biología, Universitat de Barcelona. mhernandez@ub.edu.
} 


\section{INTRODUCCIÓN}

Las variaciones estacionales reflejan las relaciones de las poblaciones con su medio ambiente, y en el caso de la especie humana los factores socioculturales constituyen un componente fundamental del ambiente en el que viven. Así pues, la organización social y el hábitat geográfico diseñan las condiciones de vida. La existencia de distintos patrones estacionales en las variables biodemográficas de la población evidencia la estructuración de la sociedad en grupos diferentes. La luz, la temperatura y la humedad siguen patrones estacionales en el ciclo anual, y las actividades sociales, rituales y religiosas también dependen de dicho ciclo, de modo que puede condicionar el comportamiento de los grupos humanos. Los ciclos estacionales pueden afectar la fisiología humana, el estado nutricional, la fecundidad, y el crecimiento, así como la morbilidad y la mortalidad (Johnston 1993).

La estacionalidad de las variables vitales consiste en su distribución no uniforme a lo largo del año, y es el resultado de las interacciones entre biología y cultura que intervienen en la diversidad de patrones biodemográficos de las poblaciones humanas. En el estudio biodemográfico del poblamiento colonizador de la Región de Magallanes se estudió la estacionalidad de las variables vitales, nacimientos y defunciones (García-Moro et al. 1995, 1997; Hernández et al. 1995, 1997), así como la de los matrimonios (García-Moro et al. 1992). El objetivo del presente trabajo es analizar la estacionalidad de estas variables a principios del siglo XXI y comparar estos resultados con los hallados durante la etapa colonizadora (18851920) (Hernández 2010) con el fin de conocer cómo los cambios en las condiciones biosociales afectan los patrones biodemográficos de la población.

\section{MATERIAL Y MÉTODOS}

En este trabajo se han utilizado los datos de las estadísticas vitales proporcionados por el Instituto Nacional de Estadística que pueden ser consultados en linea (www.ine.cl), relativos al período de 1997 hasta 2009 (excepto los años 2001, 2002 y 2004, cuyos resultados no están disponibles). Se trata de la distribución mensual de nacimientos, matrimonios y defunciones durante diez años a caballo entre el siglo pasado y el inicio del siglo XXI. Con objeto de comparar la situación actual de la población magallánica con la de la época de la colonización se han utilizado los resultados de los años 1885-1920. En el caso de los nacimientos también se ha comparado con el inicio del último cuarto del siglo XX utilizando los datos correspondientes a la provincia de Magallanes entre 1975 y 1979, mientras que en el estudio de los matrimonios también se ha comparado con la estacionalidad de otros tres períodos a lo largo del siglo XX.

La metodología estadística utilizada para el análisis de la estacionalidad ha consistido en la aplicación del test de bondad de ajuste (O'Brien \& Holbert, 1987): la distribución mensual se ha comparado con la esperada en ausencia de estacionalidad según los días de cada mes. También se han calculado los coeficientes mensuales según la metodología descrita por Henry $(1972,1983)$, de modo que el valor 100 sea el coeficiente mensual esperado en ausencia de estacionalidad, teniendo en cuenta el número de días del mes.

Con el fin de minimizar las diferencias entre las frecuencias de meses sucesivos que pueden ser debidas al artefacto de tratar un fenómeno temporal continuo a través de la discontinuidad que implica la división del año en doce meses, se han obtenido también las distribuciones de las medias móviles considerando para cada mes el promedio de su valor y los valores de los meses anterior y posterior al mismo. Estos coeficientes han sido utilizados en algunas de las representaciones gráficas.

Se ha aplicado también la metodología propuesta por Edwards (1961) para contrastar los resultados observados con el modelo teórico de una distribución armónica simple, con el fin de establecer si se ajusta a un tipo de distribución con un máximo y un mínimo equidistantes en la recurrencia anual. Reijneveld (1990) considera este método más potente que el de bondad de ajuste ya que este último puede señalar como diferencia significativa con las frecuencias teóricas cualquier alteración aleatoria, producida a veces por el artefacto experimental de dividir la secuencia temporal continua en doce clases discretas. Estas alteraciones aleatorias de las frecuencias, lejos de señalar una tendencia cíclica con significación biológica, pueden aparecer como meros resultados estadísticos sin sentido. Por ello, aunque normalmente los resultados de las pruebas de bondad de ajuste y de Edwards coinciden, en caso de discrepancia el test de Edwards puede ser más adecuado para interpretar la distribución mensual del evento. 


\section{RESULTADOS}

\section{Nacimientos}

Se ha observado estacionalidad de los nacimientos en muchas poblaciones, pero con gran diversidad de patrones. Además, para una misma población se producen cambios en su patrón estacional a lo largo del tiempo. Lam \& Miron (1991) sistematizan la mayor parte de las ideas sobre el tema y llegan a la conclusión de que no hay una explicación única y sencilla para la estacionalidad de los nacimientos. Entre los factores externos que pueden estar implicados se han citado los climáticos (temperatura, pluviosidad), el ciclo agrícola, las variables económicas y sociales (días festivos, celebración de los matrimonios, condicionantes religiosos sobre las relaciones sexuales), etc.

Prioux (1988) observó que la estacionalidad varía con la paridad y la legitimidad, y para Italia en la segunda mitad del siglo XX comprobó que las variaciones estacionales de los matrimonios podían influir en los nacimientos de las primíparas, pero esto no ocurría en Francia ni en Holanda. También observó que la tendencia a programar el embarazo era más fuerte para el segundo hijo que para los siguientes.

Cowgill (1966a) cita que a mediados del siglo XX en Europa los nacimientos tienden a aumentar a final del invierno y principio de la primavera, con un pico menor a principio del otoño. La misma autora cree que a nivel mundial la temperatura puede influir ya que observa patrones inversos entre los hemisferios (Cowgill 1966b). También advierte que la ilegitimidad, la pobreza y los conflictos pueden incrementar la estacionalidad de los nacimientos. Warren \& Tyler (1979) llegan a conclusiones parecidas.

Houidalle (1979) observó que en la Francia rural, hasta el siglo XX, los nacimientos son más frecuentes en invierno, $y$ luego las frecuencias mensuales mayores se desplazan hacia la primavera. También James (1990) cita para Europa una mayor frecuencia de nacimientos en primavera en la primera mitad del siglo XX.

En el período 1997-2009 la región chilena más austral se ha caracterizado por una estacionalidad significativa de los nacimientos. Como puede apreciarse en la Tabla 1, el período de abril a octubre muestra una frecuencia de nacimientos superior a lo esperado. La visualización de los coeficientes mensuales de las medias móviles (Fig. 1) lo evidencia claramente. Estos resultados suponen una mayor frecuencia de las concepciones entre los meses de julio y enero, de manera que en la segunda mitad del año se producen más fecundaciones. El pico de nacimientos en septiembre es el resultado de un pico de concepciones en diciembre y la primera semana de enero, que incluyen las fiestas navideñas y el inicio de los días festivos del verano. El número de nacimientos disminuye significativamente en verano a

Tabla 1. Coeficientes de estacionalidad de los nacimientos en la Región Magallánica (1997-2009).

\begin{tabular}{|l|c|c|c|c|c|c|}
\hline MES nacimiento & MES concep. & Región XII & Prov. Magallanes & $\begin{array}{c}\text { Última } \\
\text { Esperanza }\end{array}$ & $\begin{array}{c}\text { Tierra del } \\
\text { Fuego }\end{array}$ & $\begin{array}{c}\text { Antártica } \\
\text { Chilena }\end{array}$ \\
\hline Enero & Abril & 95,81 & 96,85 & 92,79 & 82,53 & 92,67 \\
\hline Febrero & Mayo & 96,76 & 97,71 & 96,71 & 79,25 & 83,20 \\
\hline Marzo & Junio & 97,46 & 99,15 & 83,47 & 107,59 & 92,67 \\
\hline Abril & Julio & 104,17 & 104,76 & 101,14 & 98,99 & 108,82 \\
\hline Mayo & Agosto & 102,20 & 101,63 & 102,11 & 106,12 & 130,58 \\
\hline Junio & Sept. & 101,45 & 99,06 & 115,15 & 108,13 & 108,82 \\
\hline Julio & Octubre & 99,00 & 99,71 & 92,37 & 103,17 & 105,31 \\
\hline Agosto & Nov. & 101,84 & 99,95 & 110,16 & 100,22 & 151,64 \\
\hline Septiembre & Dic. & 109,07 & 107,52 & 115,15 & 115,75 & 134,93 \\
\hline Octubre & Enero & 100,19 & 100,08 & 104,23 & 103,17 & 58,97 \\
\hline Noviembre & Febrero & 98,26 & 98,10 & 100,70 & 108,13 & 56,58 \\
\hline Diciembre & Marzo & 93,80 & 95,49 & 86,01 & 86,96 & 75,82 \\
\hline $\begin{array}{l}\text { TOTAL } \\
\text { nacimientos }\end{array}$ & & 22,859 & 19.000 & 2.779 & 800 & 280 \\
\hline
\end{tabular}




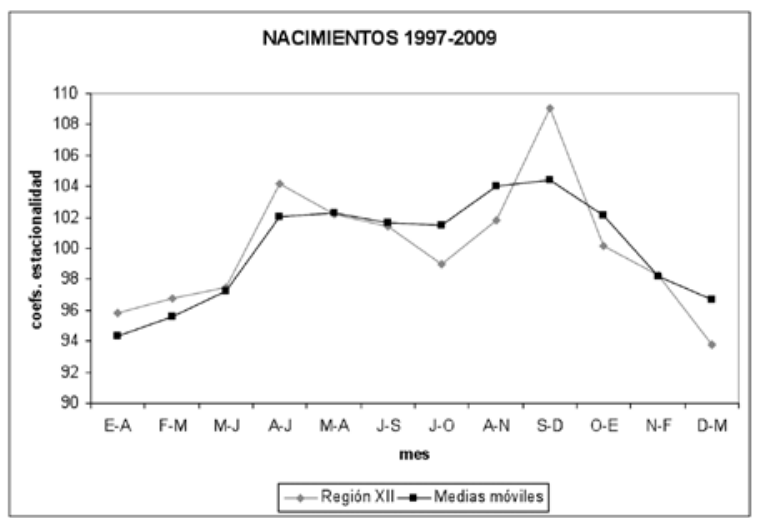

Fig. 1. Coeficientes de estacionalidad de los nacimientos en la Región XII (1997-2009).

Valores observados y medias móviles.

consecuencia de la menor frecuencia de concepciones otoñales. Aplicando el test de bondad de ajuste y el test de Edwards (Tabla 2) se comprueba la significación estadística de la estacionalidad.

Cuando se comparan las distribuciones mensuales de los nacimientos en las cuatro provincias magallánicas (Tabla 1 y Fig. 2), se observan en general unos resultados homogéneos. Como cabe esperar, la provincia de Magallanes tiene un patrón casi idéntico al del total regional debido a su mayor aportación demográfica al conjunto. La provincia de Última Esperanza presenta un número de nacimientos superior al esperado en ausencia de estacionalidad entre los meses de abril y noviembre, resultado de la mayor frecuencia de concepciones a partir de julio y hasta los meses estivales inclusive. En las provincias con menor número de habitantes las diferencias mensuales tienden a agudizarse como consecuencia de su menor tamaño demográfico. En Tierra del Fuego los resultados no resultan significativos (Tabla 2 ) debido posiblemente a este hecho. La provincia de la Antártica Chilena, con su población reproductora radicada en la isla de Navarino, presenta un número de nacimientos muy bajo para mostrar una tendencia estable pero con diferencias mensuales que dan una estacionalidad significativa; aquí los nacimientos se incrementan entre los meses de abril y septiembre en mayor medida que en el conjunto regional, resultado de la mayor proporción de concepciones en la segunda mitad del año. Los resultados de las comparaciones estadísticas entre las cuatro provincias (Tabla 3) reflejan la homogeneidad de la región ya que en ningún caso hay diferencias significativas.
Para comparar la estacionalidad de los nacimientos en la Región de Magallanes con la de la totalidad de la población chilena se han utilizado los datos de las distribuciones mensuales de nacimientos de Chile en los años 1997 y 2009. La distribución conjunta de estos dos años en representación del período estudiado muestra estacionalidad con el test de bondad de ajuste $\left(X^{2}=367,110 ; 11 \mathrm{gdl} ; \mathrm{p}<0,001\right)$ y con el de Edwards $\left(X^{2}=47,073 ; 2\right.$ gdl; $\left.p<0,001\right)$. Hay un pico de los nacimientos en el mes de septiembre, al igual que en la Región de Magallanes, pero la mayor frecuencia se presenta en el mes de enero (concepciones en abril). De manera que las distribuciones de nacimientos y concepciones en Magallanes tienen un patrón distinto al total de Chile $\left(\mathrm{X}^{2}=38,012 ; 11\right.$ gdl; $\left.\mathrm{p}<0,001\right)$ reflejando la singularidad de la región más austral en su comportamiento reproductor.

Un objetivo de este trabajo es el de conocer si se han producido cambios en los patrones de reproducción de la población actual de la Región de Magallanes en relación con los observados en la época de la colonización (1885-1920). En el estudio realizado anteriormente (Hernández et al. 1995) se observó una mayor frecuencia de nacimientos en la primavera austral (septiembre-octubre-noviembre) fruto de las concepciones estivales (diciembre-enero-febrero), siguiendo el patrón descrito para las poblaciones europeas en el siglo XX (Cowgill 1966a; Lam \& Miron 1991). Estos resultados podrían interpretarse como el mantenimiento de los patrones de origen europeo de muchas de las familias colonizadoras de la región. Los resultados de las pruebas estadísticas que verifican la existencia de un patrón estacional en el período 18851920 pueden verse en la Tabla 2.

Además, se había observado una serie de resultados interesantes con los nacimientos en el período 1885-1915 (Hernández \& García-Moro 1997): había correlación entre el número de concepciones y la temperatura mensual; pero no había relación entre la estacionalidad de los nacimientos y la profesión del padre, ni con la legitimidad. Tampoco había diferencias en la estacionalidad entre las provincias magallánicas, pero sí que había diferencias entre los nacimientos en Punta Arenas y los del resto del territorio con mayor estacionalidad que en la capital. Se observaron diferencias en la estacionalidad según la nacionalidad de los padres cuando ambos tenían la misma nacionalidad de origen. De manera que el comportamiento reproductor estacional era diverso según el origen de los progenitores 


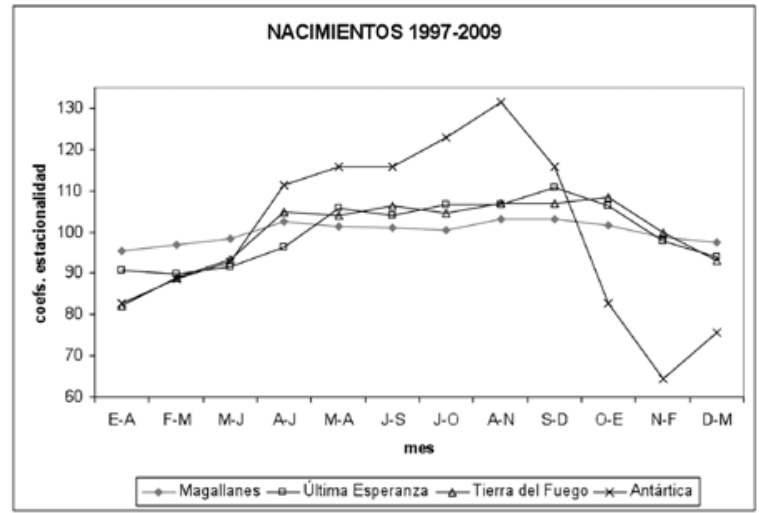

Fig. 2. Medias móviles de los coeficientes de estacionalidad de los nacimientos en las cuatro provincias magallánicas (1997-2009).

reflejando la falta de homogeneidad de la población en aquel período de asentamiento en el que se mantenía una elevada endogamia.

El comportamiento reproductor puede reflejar los cambios sociales en el devenir del proceso histórico de la población. En este sentido, en el estudio particular de la provincia de Tierra del Fuego se observó (Pascual et al. 2000) ausencia de estacionalidad entre 1890 y 1920, y también en el período 1946-1995. Solamente en el período entre 1921 y 1945 apareció estacionalidad significativa, posiblemente como reflejo de la homogeneización social de la población fruto de la edad dorada del poblamiento colonizador (Martinic 1998). En el período ahora estudiado sigue sin resultar significativa la estacionalidad de los nacimientos fueguinos, y es la única provincia magallánica en que se da este resultado (Tabla 2), aunque debe tenerse en cuenta el reducido número de nacimientos que puede condicionar el resultado de las pruebas estadísticas.

Cuando se compara la estacionalidad de los nacimientos de la población actual de la Región de Magallanes con la de la época de la colonización se observan diferencias significativas en sus patrones estacionales $\left(X^{2}=30,591 ; 11 \mathrm{gdl} ; \mathrm{p}<0,01\right)$. El máximo de nacimientos en noviembre (concepciones en febrero) del pasado se ha avanzado al mes de septiembre (concepciones en diciembre), aunque los picos de ambos períodos tengan lugar en primavera (Fig. 3).

En realidad, puede precisarse que el patrón estacional ha ido cambiando a lo largo del siglo XX, ya que se ha comparado con los datos mensuales de
Tabla 2. Valores del test de Edwards y de la bondad de ajuste para la estacionalidad de los nacimientos en la región magallánica.

\begin{tabular}{|l|c|c|}
\hline & $\begin{array}{c}\text { Test de } \\
\text { Edwards } \\
\mathrm{X}^{2}(2 \mathrm{gdl})\end{array}$ & $\begin{array}{c}\text { Test de bondad de } \\
\text { ajuste } \\
\mathrm{X}^{2}(11 \mathrm{gdl})\end{array}$ \\
\hline Región XII & $\begin{array}{c}24,105 \\
\mathrm{P}<0,001\end{array}$ & $\begin{array}{c}35,440 \\
\mathrm{P}<0,001\end{array}$ \\
\hline Provincia & 9,610 & 19,264 \\
Magallanes & $\mathrm{P}<0,01$ & $\mathrm{P}=0,057$ \\
\hline Última & 15,522 & 27,407 \\
Esperanza & $\mathrm{P}<0,001$ & $\mathrm{P}=0,004$ \\
\hline Tierra del Fuego & 5,251 & 9,164 \\
\hline Antártica Chilena & $\mathrm{P}>0,05$ & $\mathrm{P}=0,607$ \\
\hline & $\mathrm{P}<0,01$ & 22,336 \\
\hline Región XII & 18,566 & $\mathrm{P}=0,022$ \\
1885-1920 & $\mathrm{P}<0,001$ & $\mathrm{P}<0,001$ \\
\hline Provincia & 28,341 & 53,526 \\
Magallanes & $\mathrm{P}<0,001$ & $\mathrm{P}<0,001$ \\
1975-1979 & & \\
\hline
\end{tabular}

nacimientos en la provincia de Magallanes (procedentes del Registro Civil de Punta Arenas) del período 19751979, que muestran también patrón estacional (la significación estadística puede verse en la Tabla 2), con un pico importante de nacimientos en septiembre, pero con una distribución mensual estadísticamente distinta de la actual, tanto si se compara con los datos actuales de toda la región $\left(\mathrm{X}^{2}=35,764 ; 11 \mathrm{gdl} ; \mathrm{p}<\right.$ $0,001)$ como con los datos actuales de la provincia de Magallanes ( $\mathrm{X}^{2}=36,366 ; 11 \mathrm{gdl} ; \mathrm{p}<0,001$ ). También difiere el patrón estacional de esta provincia en 1975-1979 con el patrón regional de la época de la colonización $\left(X^{2}=29,863 ; 11\right.$ gdl; $\left.p<0,01\right)$. En resumen, se ha comprobado que el desplazamiento del pico de nacimientos de noviembre a septiembre ya era vigente al iniciarse el último cuarto del siglo XX.

\section{Matrimonios}

La estacionalidad de los matrimonios tiene un claro determinismo socio-económico que puede relacionarse con los ciclos de la producción y la estacionalidad de la actividad laboral. En la época de la colonización regional de Magallanes se observó (García-Moro et al. 1992) una estacionalidad claramente condicionada por el ciclo anual de la actividad ganadera, 
Tabla 3. Valores del test de homogeneidad entre las provincias magallánicas para la estacionalidad de los nacimientos (1997-2009).

\begin{tabular}{|l|c|c|c|}
\hline & $\begin{array}{c}\text { Última } \\
\text { Esperanza }\end{array}$ & $\begin{array}{c}\text { Tierra del } \\
\text { Fuego }\end{array}$ & $\begin{array}{c}\text { Antártica } \\
\text { Chilena }\end{array}$ \\
\hline $\begin{array}{l}\text { Provincia } \\
\text { Magallanes }\end{array}$ & $\begin{array}{c}17,710 \\
\mathrm{P}=0,088\end{array}$ & $\begin{array}{c}6,463 \\
\mathrm{P}=0,840\end{array}$ & $\begin{array}{c}19,593 \\
\mathrm{P}=0,052\end{array}$ \\
\hline $\begin{array}{l}\text { Última } \\
\text { Esperanza }\end{array}$ & & $\begin{array}{c}7,380 \\
\mathrm{P}=0,767\end{array}$ & $\begin{array}{c}15,655 \\
\mathrm{P}=0,154\end{array}$ \\
\hline $\begin{array}{l}\text { Tierra del } \\
\text { Fuego }\end{array}$ & & & $\begin{array}{c}15,116 \\
\mathrm{P}=0,177\end{array}$ \\
\hline
\end{tabular}

de modo que la mayor frecuencia de matrimonios se producía a final del otoño y durante el invierno (con los valores más elevados en los meses de mayo, junio y julio) cuando menor era la actividad laboral (Tabla 4).

Muy distinta es la situación actual (1997-2009), en la que son los meses veraniegos los que presentan la mayor celebración de matrimonios con un pico importante en febrero del coeficiente estacional (Tabla 4 y Figura 4), mientras que entre marzo y noviembre los matrimonios resultan menos frecuentes de lo esperado en ausencia de estacionalidad. La interpretación de estos resultados debe considerar el hecho del período vacacional como condicionante del mayor número de matrimonio. Los resultados de los coeficientes de estacionalidad pueden verse en la Tabla 4, y los resultados de los tests de estacionalidad se muestran en la Tabla 5.

A efectos comparativos, se ha analizado también la distribución mensual de las celebraciones

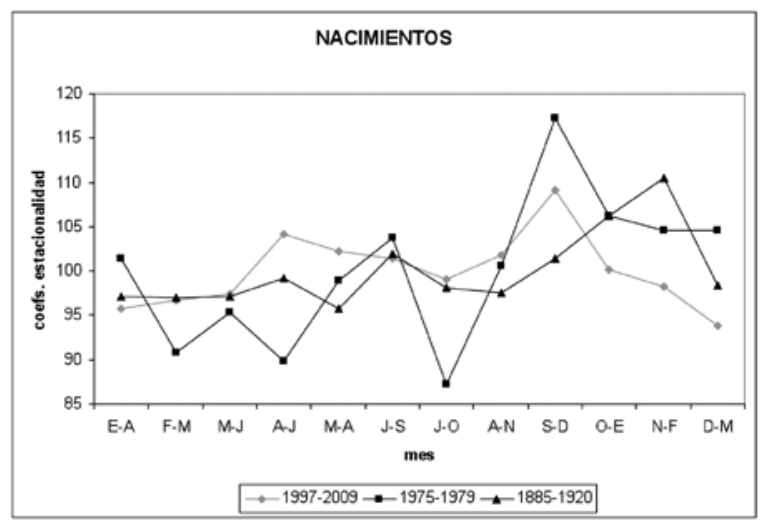

Fig. 3. Coeficientes de estacionalidad de los nacimientos en la región magallánica en tres períodos. matrimoniales con los datos de los años 1997 y 2009 del total de Chile. La distribución de Chile resulta estacional (test de bondad de ajuste: $\mathrm{X}^{2}=5336,864 ; 11 \mathrm{gdl}$; $\mathrm{P}$ $<0,001$ ) y sinusoidal (test de Edwards: $\mathrm{X}^{2}=3825 ; 2$ gdl; $\mathrm{P}<0,001)$. En el conjunto de la población chilena los matrimonios se celebran más frecuentemente entre los meses de octubre y abril, presentándose un pico importante en febrero $(148,5)$ similar al observado en la región magallánica $(147,6)$. De igual modo, el mes con menor frecuencia es el de junio (74,4 en el total chileno, y 77,9 en la Región XII). Pero la distribución mensual se presenta más concentrada en los meses estivales en la población magallánica, de modo que sus diferencias con el total chileno resultan significativas $\left(X^{2}=96,516 ; 11\right.$ gdl; $P<0,001$ ).

Las diferencias entre la situación actual de la estacionalidad matrimonial y la registrada en la época de la colonización son muy evidentes (Tabla 4 y Fig. 5) como consecuencia del cambio del patrón de mayor frecuencia invernal al de mayor frecuencia estival. Ya que se disponía de datos de la provincia de Magallanes de algunos períodos del siglo XX, obtenidos en el Registro Civil de Punta Arenas, se han analizado también con el objetivo de intentar conocer cómo se ha realizado el cambio en el patrón estacional. Las distribuciones de los coeficientes estacionales de los períodos 1946-55, 1964-73 y 1983-92 pueden verse también en la Tabla 4. En todos ellos hay estacionalidad significativa (Tabla 5).

En los cinco períodos analizados las distribuciones mensuales de los matrimonios resultan ser distintas estadísticamente (Tabla 6), pero se puede observar bien la secuencia temporal del cambio. Los valores del test de homogeneidad entre los períodos reflejan la mayor

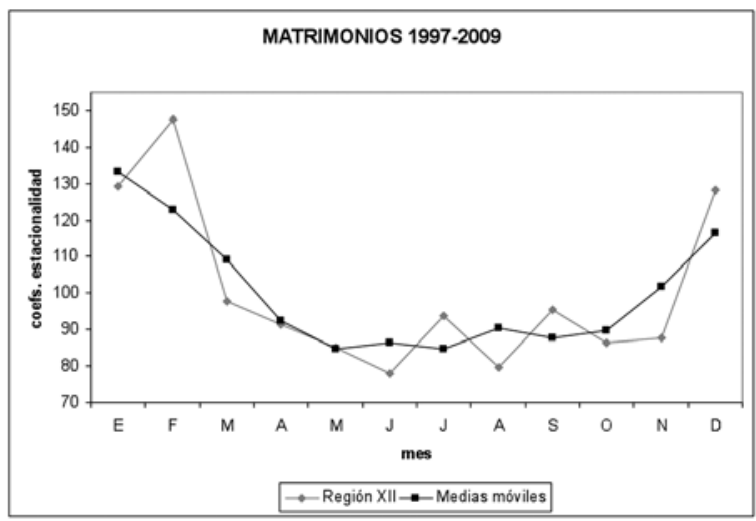

Fig. 4. Coeficientes de estacionalidad de los matrimonios en la Región XII (1997-2009).

Valores observados y medias móviles. 
Tabla 4. Coeficientes de estacionalidad de los matrimonios en la región magallánica en distintos períodos.

\begin{tabular}{|l|c|c|c|c|c|}
\hline $\begin{array}{l}\text { MES } \\
\text { matrimonio }\end{array}$ & $\begin{array}{c}\text { Región XII } \\
1885-1920\end{array}$ & $\begin{array}{c}\text { Provincia } \\
\text { Magallanes } \\
1946-1955\end{array}$ & $\begin{array}{c}\text { Provincia } \\
\text { Magallanes } \\
1964-1973\end{array}$ & $\begin{array}{c}\text { Provincia } \\
\text { Magallanes } \\
1983-1992\end{array}$ & $\begin{array}{c}\text { Región XII } \\
1997-2009\end{array}$ \\
\hline Enero & 75,73 & 87,03 & 112,92 & 113,81 & 129,23 \\
\hline Febrero & 106,24 & 97,52 & 109,47 & 115,03 & 147,60 \\
\hline Marzo & 90,44 & 88,34 & 107,42 & 100,60 & 97,76 \\
\hline Abril & 106,06 & 91,29 & 101,26 & 94,11 & 91,57 \\
\hline Mayo & 117,34 & 100,66 & 91,32 & 86,31 & 84,92 \\
\hline Junio & 136,16 & 121,63 & 96,19 & 95,80 & 77,94 \\
\hline Julio & 122,61 & 116,13 & 93,87 & 87,54 & 93,71 \\
\hline Agosto & 106,53 & 103,02 & 82,68 & 84,27 & 79,65 \\
\hline Septiembre & 102,91 & 112,96 & 111,41 & 96,08 & 95,38 \\
\hline Octubre & 73,79 & 92,54 & 86,02 & 91,89 & 86,33 \\
\hline Noviembre & 74,24 & 77,47 & 80,77 & 97,06 & 87,57 \\
\hline Diciembre & 87,94 & 111,41 & 126,67 & 137,50 & 128,35 \\
\hline $\begin{array}{l}\text { TOTAL } \\
\text { matrimonios }\end{array}$ & 4243 & 4495 & 5997 & 8649 & 6686 \\
\hline
\end{tabular}

o menor distancia temporal entre ellos, de modo que las diferencias estadísticas más importantes se dan entre la época de colonización y la actual. En cambio, entre los períodos 1964-73 y 1983-92 se producen las menores diferencias. También resultan relativamente pequeñas las diferencias entre el período más antiguo y el de 1946-55, ya que en ambos se dan los valores más elevados en los meses de invierno y es después cuando cambia la tendencia para pasar a mayores frecuencias en verano.

Si la interpretación de las causas de la estacionalidad matrimonial relacionada con la actividad ganadera en el período 1885-1920 estaba fundamentada, el cambio observado después de 1955 podría estar

Tabla 5. Valores del test de Edwards y de la bondad de ajuste para la estacionalidad de los matrimonios en la región magallánica en distintos períodos.

\begin{tabular}{|l|c|c|}
\hline & $\begin{array}{c}\text { Test de Edwards } \\
\mathrm{X}^{2}(2 \text { gdl })\end{array}$ & $\begin{array}{c}\text { Test de bondad de } \\
\text { ajuste } \\
\mathrm{X}^{2}(11 \mathrm{gdl})\end{array}$ \\
\hline $\begin{array}{l}\text { Región XII } \\
\text { 1885-1920 }\end{array}$ & $\begin{array}{c}126,402 \\
\mathrm{P}<0,001\end{array}$ & $\begin{array}{c}157,117 \\
\mathrm{P}<0,001\end{array}$ \\
\hline $\begin{array}{l}\text { Provincia de } \\
\text { Magallanes } \\
\text { 1946-1955 }\end{array}$ & $\begin{array}{c}32,135 \\
\mathrm{P}<0,001\end{array}$ & $\begin{array}{c}74,190 \\
\mathrm{P}<0,001\end{array}$ \\
\hline $\begin{array}{l}\text { Provincia de } \\
\text { Magallanes } \\
\text { 1964-1973 }\end{array}$ & $\begin{array}{c}28,143 \\
\mathrm{P}<0,001\end{array}$ & $\begin{array}{c}108,155 \\
\mathrm{P}<0,001\end{array}$ \\
\hline $\begin{array}{l}\text { Provincia de } \\
\text { Magallanes }\end{array}$ & $\begin{array}{c}98,618 \\
\text { 1983-1992 }\end{array}$ & $\begin{array}{c}186,170 \\
\mathrm{P}<0,001\end{array}$ \\
\hline $\begin{array}{l}\text { Región XII } \\
\text { 1997-2009 }\end{array}$ & $\begin{array}{c}158,704 \\
\mathrm{P}<0,001\end{array}$ & $\begin{array}{c}302,402 \\
\mathrm{P}<0,001\end{array}$ \\
\hline
\end{tabular}

relacionado con cambios en la actividad económica que afectarían al comportamiento social. Ha sido descrito en otras poblaciones (Esparza et al. 2004) el hecho del cambio en la estacionalidad matrimonial cuando la economía se diversifica y se producen cambios importantes en las faenas del campo. En este sentido, aquí debemos tener en cuenta que en la región magallánica, en el intervalo entre 1955 y 1964, la producción de hidrocarburos hizo disminuir la importancia relativa de la actividad ganadera (Martinic 1992). Así pues, la vida social se habría visto modificada por la pérdida de importancia relativa de dicha actividad.

\section{Defunciones}

La mortalidad general también suele presentar distribuciones estacionales en las poblaciones humanas dependiendo de la incidencia de la estacionalidad de algunas de las causas más importantes. En la Tablas 7 y la Fig. 6 se muestran las distribuciones de los coeficientes mensuales de estacionalidad de las defunciones en la región magallánica, y en la Tabla 8 los resultados de las pruebas de bondad de ajuste y del test de Edwards, para el período de la colonización y en la actualidad. Como puede apreciarse, en ambos períodos se presenta estacionalidad aunque con una intensidad mucho mayor en el período 1885-1920 en el que se alcanza un pico de 137,6 en el mes de septiembre. Por otro lado, las distribuciones de ambos períodos son significativamente distintas ( $\left.X^{2}=165,443 ; 11 \mathrm{gdl} ; \mathrm{P}<0,001\right)$ ya que en el primer período el mayor número de defunciones se 


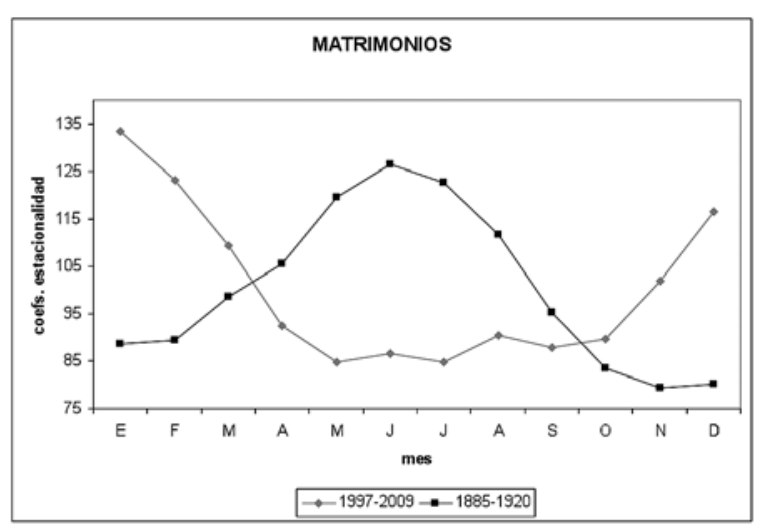

Fig. 5. Contraste entre la estacionalidad matrimonial en la Región Magallánica actual con la del período de colonización.

produce en los meses de primavera (García-Moro et al. 1995), y en la actualidad ello sucede en invierno (con un pico de 110,3 en julio). Estas diferencias son el resultado del cambio secular en las condiciones de vida en la región magallánica.

La población chilena actual se caracteriza por presentar estacionalidad en la distribución de su mortalidad general $\left(X^{2}=913,600 ; 11\right.$ gdl; $\left.P<0,001\right)$ que se puede asimilar a una distribución sinusoidal $\left(\mathrm{X}^{2}\right.$ =910,930; 2 gdl; $\mathrm{P}<0,001)$. Cuando se compara la mortalidad de la población actual magallánica con la del conjunto chileno (años 1997 y 2009) se observan diferencias significativas aunque relativamente pequeñas $\left(\mathrm{X}^{2}=21,198 ; 11 \mathrm{gdl} ; \mathrm{P}=0,031\right)$ ya que el total chileno tiene valores algo mayores en los meses en que las defunciones superan los valores esperados en ausencia de estacionalidad (de mayo a octubre en Chile con un pico, también en julio, de 113,6).

Tabla 6. Valores del test de homogeneidad entre distintos períodos para la estacionalidad de los matrimonios en la región magallánica.

\begin{tabular}{|l|c|c|c|c|}
\hline & $\begin{array}{c}\text { P. Magallanes } \\
1946-1955\end{array}$ & $\begin{array}{c}\text { P. Magallanes } \\
1964-1973\end{array}$ & $\begin{array}{c}\text { P. Magallanes } \\
1983-1992\end{array}$ & $\begin{array}{c}\text { Región XII } \\
1997-2009\end{array}$ \\
\hline $\begin{array}{l}\text { Región XII } \\
1885-1920\end{array}$ & $\begin{array}{c}36,727 \\
\mathrm{P}<0,001\end{array}$ & $\begin{array}{c}144,051 \\
\mathrm{P}<0,001\end{array}$ & $\begin{array}{c}217,274 \\
\mathrm{P}<0,001\end{array}$ & $\begin{array}{c}263,246 \\
\mathrm{P}<0,001\end{array}$ \\
\hline $\begin{array}{l}\text { P. Magallanes } \\
\text { 1946-1955 }\end{array}$ & & $\begin{array}{c}67,916 \\
\mathrm{P}<0,001\end{array}$ & $\begin{array}{c}110,146 \\
\mathrm{P}<0,001\end{array}$ & $\begin{array}{c}170,487 \\
\mathrm{P}<0,001\end{array}$ \\
\hline $\begin{array}{l}\text { P. Magallanes } \\
\text { 1964-1973 }\end{array}$ & & & $\begin{array}{c}24,989 \\
\mathrm{P}=0,009\end{array}$ & $\begin{array}{c}57,847 \\
\mathrm{P}<0,001\end{array}$ \\
\hline $\begin{array}{l}\text { P. Magallanes } \\
\text { 1983-1992 }\end{array}$ & & & & $\begin{array}{c}50,418 \\
\mathrm{P}<0,001\end{array}$ \\
\hline
\end{tabular}

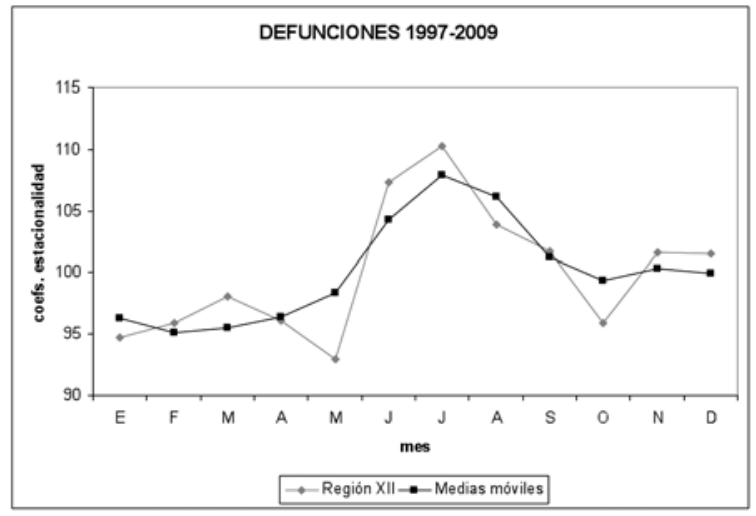

Fig. 6. Coeficientes de estacionalidad de las defunciones en la Región XII (1997-2009). Valores observados y medias móviles.

\section{CONCLUSIONES}

Los eventos vitales presentan estacionalidades estadísticamente significativas en la Región Magallánica en la actualidad. Los nacimientos son más frecuentes entre los meses de abril a octubre, con un máximo en septiembre, lo que indica un aumento de las concepciones en las fiestas navideñas y el inicio del verano, no observándose diferencias significativas del patrón estacional entre las cuatro provincias magallánicas. La estacionalidad de los matrimonios se caracteriza por

Tabla 7. Coeficientes de estacionalidad de las defunciones en la región magallánica en los períodos $1885-1920$ y 1997-2009.

\begin{tabular}{|l|c|c|}
\hline MES defunción & $\begin{array}{c}\text { Región XII } \\
1885-1920\end{array}$ & $\begin{array}{c}\text { Región XII } \\
1997-2009\end{array}$ \\
\hline Enero & 94,04 & 94,74 \\
\hline Febrero & 81,60 & 95,91 \\
\hline Marzo & 78,96 & 98,10 \\
\hline Abril & 75,74 & 96,10 \\
\hline Mayo & 81,31 & 93,00 \\
\hline Junio & 88,41 & 107,29 \\
\hline Julio & 97,58 & 110,30 \\
\hline Agosto & 105,00 & 103,83 \\
\hline Septiembre & 137,61 & 101,76 \\
\hline Octubre & 123,50 & 95,86 \\
\hline Noviembre & 122,75 & 101,63 \\
\hline Diciembre & 113,49 & 101,47 \\
\hline $\begin{array}{l}\text { TOTAL } \\
\text { defunciones }\end{array}$ & 10005 & 9465 \\
\hline
\end{tabular}


Tabla 8. Valores del test de Edwards y de la bondad de ajuste para la estacionalidad de las defunciones en los períodos $1885-1920$ y $1997-2009$.

\begin{tabular}{|l|c|c|}
\hline & $\begin{array}{c}\text { Test de Edwards } \\
\mathrm{X}^{2}(2 \text { gdl })\end{array}$ & $\begin{array}{c}\text { Test de bondad de } \\
\text { ajuste } \\
\mathrm{X}^{2}(11 \text { gdl })\end{array}$ \\
\hline $\begin{array}{l}\text { Región XII } \\
1885-1920\end{array}$ & $\begin{array}{c}368,264 \\
\mathrm{P}<0,001\end{array}$ & $\begin{array}{c}379,030 \\
\mathrm{P}<0,001\end{array}$ \\
\hline $\begin{array}{l}\text { Región XII } \\
1997-2009\end{array}$ & $\begin{array}{c}14,717 \\
\mathrm{P}<0,001\end{array}$ & $\begin{array}{c}24,688 \\
\mathrm{P}=0,010\end{array}$ \\
\hline
\end{tabular}

una mayor frecuencia en los meses veraniegos con un máximo en febrero. También las defunciones presentan estacionalidad con mayor incidencia en invierno y un máximo en el mes de julio.

Otra conclusión es que se han observado diferencias de los patrones estacionales de la región chilena más austral con los de la población chilena total, reflejando la diferenciación regional que singulariza a la región magallánica. Por último, ha podido evidenciarse con claridad, cómo los cambios socioeconómicos acaecidos en la región han modificado los patrones estacionales de las tres variables estudiadas, ya que los resultados actuales muestran diferencias significativas con los de la época de la colonización.

\section{BIBLIOGRAFÍA}

Cowgill, U.M. (1966a). The season of birth in man. Man, 1, 232-240. Cowgill, U.M. (1966b). Season of birth in man. Contemporary situation with special reference to Europe and the Southern hemisphere. Ecology, 47, 614-623.

Edwards, J.H. (1961). The recognition and estimation of cyclic trends. Annals of Human Genetics London, 25, 83-87.

Esparza, M., García-Moro, C., \& Hernández, M. (2004). Biodemografia dels matrimonis del delta de l'Ebre. Recerca, 8, 69-117.

García-Moro, C., \& Hernández, M. (1997). Patrones de mortalidad en la población chilena de Tierra del Fuego. Revista Española de Antropología Biológica, 18, 231-245.

García-Moro, C., Hernández, M., \& Martinic, M. (1992). Estacionalidad de los matrimonios en Magallanes, Chile (1885-1920). Anales del Instituto de la Patagonia, Serie Ciencias Humanas, 21, 47-61.

García-Moro, C. Hernández, M., \& Martinic, M. (1995). Estructura de la mortalidad infantil en la población colonizadora de Magallanes (1885-1920). Anales del Instituto de la Patagonia, Serie Ciencias Humanas, 23, 67-74.

Henry, L. (1972). Démographie, analyse et modèles. Paris: Larousse. Henry, L. (1983). Manual de demografía histórica. Barcelona: Crítica.

Hernández, M. (2010). Migración, colonización y patrones de poblamiento en Cataluña y el Cono Sur americano en los siglos XIX y XX. Revista Española de Antropología Física, 31, 123-148.

Hernández, M., \& García-Moro, C. (1997). Estacionalidad de los nacimientos en la población colonizadora de la Región Magallánica (Patagonia, Chile). Estudios de Antropología Biológica, 8, 243-257.

Hernández, M., García-Moro, C., \& Martinic, M. (1995). Reproducción de la población colonizadora de la Patagonia chilena. Anales del Instituto de la Patagonia, Serie Ciencias Humanas, 23, 53-65.

Houdaille, J. (1979). Mouvement saisonnier des conceptions en France de 1740 à 1829. Population, 34(2), 452-457.

James, W.H. (1990). Seasonal variation in human births. Journal of Biosocial Science, 22, 113-119.

Johnston, F.E. (1993). Seasonality and human biology. In: S.J. Ulijascek, \& S.S. Strickland (Eds.), Seasonality and Human Ecology (pp. 5-16). Cambridge: Cambridge University Press.

Lam, D.A., \& Miron, J.A. (1991). Seasonality of births in human populations. Social Biology, 38, 51-78.

Martinic, M. (1992). Historia de la Región Magallánica. Santiago de Chile: Alfabeta Imp.

Martinic, M. (1998). La Tierra de los Fuegos. Porvenir: Municipalidad de Porvenir.

O'Brien, K.F., \& Holbert, D. (1987): Note of the choice of stastistic for testing hypotheses regarding seasonality. American Journal of Physical Anthropology, 75, 523-524.

Pascual, J., García-Moro, C., \& Hernández, M. (2000). Nonseasonality of births in Tierra del Fuego (Chile). Annals of Human Biology, 27, 517-524.

Prioux, F. (1988). Mouvement seasonnier des naissances: Influence du rang et de la légitimité dans quelques pays d'Europe occidentale. Population, 43(3), 587-610.

Reijneveld, S.A. (1990). The choice of a statistic for testing hypotheses regarding seasonality. American Journal of Physical Anthropology, 83, 181-184.

Warren, C.W., \& Tyler, C.W. (1979). Social status and season of birth: a study of a metropolitan area in the Southeastern United States. Social Biology, 26(4), 275-288. 
